\title{
Connections with Symplectic Structures
}

\author{
A. K. M. Nazimuddin'1, Md. Showkat Ali² \\ ${ }^{1}$ Department of Electronics and Communications Engineering, East West University, Dhaka, Bangladesh \\ ${ }^{2}$ Department of Applied Mathematics, University of Dhaka, Dhaka, Bangladesh \\ Email: nazimuddin@ewubd.edu,msa317@yahoo.com
}

How to cite this paper: Nazimuddin, A.K.M. and Ali, Md.S. (2016) Connections with Symplectic Structures. American Journal of Computational Mathematics, 6, 313-319. http://dx.doi.org/10.4236/ajcm.2016.64032

Received: October 6, 2016

Accepted: December 18, 2016

Published: December 21, 2016

Copyright $\odot 2016$ by authors and Scientific Research Publishing Inc. This work is licensed under the Creative Commons Attribution International

License (CC BY 4.0).

http://creativecommons.org/licenses/by/4.0/

\begin{abstract}
A charming feature of symplectic geometry is that it is at the crossroad of many other mathematical disciplines. In this article we review the basic notions with examples of symplectic structures and show the connections of symplectic geometry with the various branches of differential geometry using important theorems.
\end{abstract}

\section{Keywords}

Connection, Differential Geometry, Symplectic Geometry

\section{Introduction}

Symplectic geometry originated in Hamiltonian dynamics. Symplectic geometry is the study of symplectic structures. These are certain topological structures, but these can only exist on even dimensional manifolds. Since symplectic structures are purely topological structures, they do not depend on any metric structure of the underlying space. In the earlier work, Nazimuddin and Rifat (2014) developed a comparison between symplectic and Riemannian geometry [1]. After summarizing the basic definitions, examples and facts concerning symplectic geometry, this article will proceed to discuss the connections between symplectic geometry and contact geometry, Riemannian geometry, Kähler geometry.

\section{Basic Concepts with Examples}

Let $M$ be a even dimensional smooth closed manifold, that is a compact smooth manifold without boundary. A symplectic structure $\omega$ on $M$ is a closed $(\mathrm{d} \omega=0)$, nondegenerate $\left(\omega^{n}=\omega \wedge \cdots \wedge \omega \neq 0\right)$ smooth 2 -form. The nondegeneracy condition is equivalent to the fact that $\omega$ induces an isomorphism. In symplectic geometry, conformal changes to $\omega$ (i.e., multiplying by $g$ ) would usually force $\mathrm{d}(g \omega) \neq 0$. 
Example 2.1. The standard symplectic structure on $\mathbb{R}^{2 n}$ is given by

$$
\omega_{0}=\sum_{i=1}^{n} \mathrm{~d} x_{i} \wedge \mathrm{d} y_{i}
$$

where $\left(x_{1}, x_{2}, \cdots, x_{n}, y_{1}, y_{2}, \cdots, y_{n}\right)$ are the coordinates of $\mathbb{R}^{2 n}$. It is clear that $\omega_{0}$ is closed.

Example 2.2. All manifolds are not symplectic. For instance, $S^{4}$ is not. If $\omega_{0}$ is a symplectic form on $S^{4}$, then $\omega_{0}$ is exact, since the second homology class of $S^{4}$ vanishes [2]. In other words, since $\omega_{0}$ is a closed 2-form $\omega_{0}=\mathrm{d} \alpha_{0}$, for some 1-form $\alpha_{0}$ and $\mathrm{d}\left(\omega_{0} \wedge \alpha_{0}\right)=\omega_{0} \wedge \omega_{0}$. Since $\omega_{0} \wedge \omega_{0}$ is a volume form on $S^{4}$, Stokes theorem implies that

$$
\int_{S^{4}} \omega_{0} \wedge \omega_{0}=\int_{\delta S^{4}} \omega_{0} \wedge \alpha_{0} \neq 0
$$

Since $S^{4}$ has no boundary, the last integral vanishes and $\omega_{0}$ can have no symplectic form.

\section{Local Theory}

The natural equivalence between symplectic structures is symplectomorphism. Two symplectic structures $\omega_{1}$ and $\omega_{2}$ on manifolds $M_{1}$ and $M_{2}$, respectively, are symplectomorphic if there exists a diffeomorphism $\varphi: M_{1} \rightarrow M_{2}$ satisfying $\varphi^{*}\left(\omega_{1}\right)=\omega_{2}$. All symplectic structures are locally symplectomorphic. In consequence, there are no local invariants in symplectic geometry according to the following theorems. In particular case, We have Darboux's theorem which states that, all symplectic structures on a $2 n$ dimensional manifold are locally symplectomorphic to the standard structure on $\mathbb{R}^{2 n}$.

Theorem 3.1 (Darboux's theorem) Let $M$ be a manifold of dimension $2 n$ with a closed non-degenerate 2-form $\omega_{0}$. For any point $p$ on a symplectic manifold, there $e^{x}$ ists a chart $U$ with local coordinates $\left(x_{1}, x_{2}, \cdots, x_{n}, y_{1}, y_{2}, \cdots, y_{n}\right)$, such that on $U$

$$
\omega_{0}=\sum_{i=1}^{n} \mathrm{~d} x_{i} \wedge \mathrm{d} y_{i}
$$

Thus locally all symplectic structures are symplectomorphic to Example 2.1.

Theorem 3.2 (Weinstein's Theorem) If a submanifold $L$ of a symplectic manifold $(M, \omega)$, then there exists a neighborhood of $L$ which is symplectomorphic to a neighborhood of the zero section in the cotangent bundle $T^{*} L$.

Furthermore symplectic structures are "local in time". That is symplectic deformations of symplectic structures do not produce new symplectic structures.

Theorem 3.3 (Moser's theorem) Let $M$ be a closed manifold and $\omega_{p} t \in[0,1]$ is a family of cohomologous symplectic forms on $M$ then there is an isotopy $\varphi_{t}$ with $\varphi_{0}=i d$ such that $\varphi_{t}^{*}\left(\omega_{t}\right)=\omega_{0}$ for all $t$.

In particular, on a symplectic manifold all deformations of symplectic structures come from diffeomorphisms of the underlying manifold. The theorem is not true if the symplectic structures do not agree off of a compact set. 


\section{Existence and Classification}

If a symplectic vector bundle is a pair $(E, \omega)$ over a smooth manifold $M$ of rank $2 n$, where $E \rightarrow M$ is a real vector bundle, then $\omega_{q}$ (skew-symmetric and non-degenerate) is a symplectic form on each fiber $E_{q}$, depending smoothly on $q$. Each of the following two characteristics is equivalent to the existence of a symplectic structure (a) the existence of a reduction of the structure group of $E$ from general linear group $G L(2 n)$ to symplectic group $\operatorname{Sp}(2 n, \mathbb{R})$ and (b) the existence of an (almost) complex structure on $E: J \in \operatorname{End}(E)$ such that $J^{2}=-I d$.

Now we discuss some recent results on the existence of symplectic structures on both open and closed manifolds. The existence problem of symplectic structures on even dimensional closed manifolds is quite difficult. However, Gromov has shown that symplectic structures on open manifolds obey an $h$-principle rule. As the existence problem of symplectic structures is based on a differential equation, but it can be reduced to a differential inequality and then solved by the $h$-principle.

Theorem 4.1 (Gromov's Theorem) Every $2 \mathrm{n}$ dimensional manifold $M$ with almost symplectic structure is homotopic through almost symplectic structures to a symplectic structure, if $M$ is open.

If the manifolds are closed, then the existence problem is much more subtle. Often there are no h-principle rules. The following result was obtained using Seiberg-Witten theory:

Theorem 4.2 (Taubes Theorem) The connected sum of an odd number of copies of $\mathbb{C P}^{2}$ does not admit a symplectic structure (even though it admits an almost symplectic structure and a cohomology class $\beta \in H^{2}(M)$ such that $\left.\beta^{2} \neq 0\right)$.

In higher dimensions the uniqueness problem for symplectic forms on closed manifolds does not reduce to topological obstruction theory. There is often a dramatic difference between the space of non-degenerate two-forms and the space of symplectic forms [3].

\section{Connections with Contact Geometry}

The even dimensional analogue theory to contact geometry is symplectic geometry. In general, contact manifolds come naturally as boundaries of symplectic manifolds. Also a contact manifold by symplectic means by looking at its symplectization [4] [5].

Consider $(X, \omega)$ be a symplectic manifold. A vector field $v$ satisfying

$$
L_{v} \omega=\omega
$$

where $L_{v} \omega$ is the Lie derivative of $\omega$ in the direction of $v$, is called a symplectic dilation. A compact hypersurface $M$ in $(X, \omega)$ is said to have contact type if there exists a symplectic dilation $v$ in a neighborhood of $M$ that is transverse to $M$. Given a hypersurface $M$ in $(X, \omega)$ the characteristic line field $L M$ in the tangent bundle of $M$ is the symplectic complement of $T M$ in $T X$. (Since $M$ is codimension one it is coisotropic and thus the symplectic complement lies in $T M$ and is one dimensional.)

Theorem 5.1. Let $M$ be a compact hypersurface in a symplectic manifold $(X, \omega)$ and 
denote the inclusion map $i: M \rightarrow X$. Then $M$ has contact type if and only if there exists a 1-form $\alpha$ on $M$ such that $\mathrm{d} \alpha=i^{*} \omega$ and the form $\alpha$ is never zero on the characteristic line field.

If $M$ is a hypersurface of contact type, then the 1 -form $\alpha$ is obtained by contracting the symplectic dilation $v$ into the symplectic form: $\alpha=l_{v} \omega$. It is easy to verify the 1 -form $\alpha$ is a contact from on $M$. Thus a hypersurface of contact type in a symplectic manifold inherits a co-oriented contact structure.

Given a co-orientable contact manifold $(M, \xi)$ its symplectization Symp $(M, \xi)=(X, \omega)$ is constructed as follows. The manifold $X=M \times(0, \infty)$ and given a global contact form $\alpha$ for $\xi$ the symplectic form is $\omega=\mathrm{d}(t \alpha)$, where $t$ is the coordinate on $\mathbb{R}$.

Example 5.2. The symplectization of the standard contact structure on the unit cotangent bundle is the standard symplectic structure on the complement of the zero section in the cotangent bundle.

The symplectization is independent of the choice of contact from $\alpha$. To see this fix a co-orientation for $\xi$ and note the manifold $X$ can be identified (in may ways) with the subbundle of $T^{*} M$ whose fiber over $x \in M$ is $\left\{\beta \in T_{x}^{*} M: \beta\left(\xi_{x}\right)=0\right.$ and $\beta>0$ on vectors positively transverse to $\left.\xi_{x}\right\}$ and restricting $\mathrm{d} \lambda$ the this subspace yields a symplectic form $\omega$, where $\lambda$ is the Liouville form on $T^{*} M$. A choice of contact form $\alpha$ fixes an identification of $X$ with the subbundle of $T^{*} M$ under which $\mathrm{d}(t \alpha)$ is taken to $\mathrm{d} \lambda$.

The vector field $v=\frac{\partial}{\partial t}$ on $(X, \omega)$ is a symplectic dilation that is transverse to $M \times\{1\} \subset X$. Clearly $\left.l_{v} \omega\right|_{M \times\{1\}}=\alpha$. Thus we see that any co-orientable contact manifold can be realized as a hypersurface of contact type in a symplectic manifold. In summary we have the following theorem.

Theorem 5.3. If $(M, \xi)$ is a co-oriented contact manifold, then there is a symplectic manifold $\operatorname{Symp}(M, \xi)$ in which $M$ sits as a hypersurface of contact type. Moreover, any contact form a for $\xi$ gives an embedding of $M$ into $\operatorname{Symp}(M, \xi)$ that realizes $M$ as a hypersurface of contact type.

We also note that all the hypersurfaces of contact type in $(X, \omega)$ look locally, in $X$, like a contact manifold sitting inside its symplectification.

Theorem 5.4. Given a compact hypersurface $M$ of contact type in a symplectic manifold $(X, \omega)$ with the symplectic dilation given by $v$ there is a neighborhood of $M$ in $X$ symplectomorphic to a neighborhood of $M \times\{1\}$ in $\operatorname{Symp}(M, \xi)$ where the symplectization is identified with $M \times(0, \infty)$ using the contact form $\alpha=\left.l_{v} \omega\right|_{M}$ and $\xi=\operatorname{ker} \alpha$.

The following proposition shows how symplectic structures can be generated from contact structures.

Proposition 5.5. [6] Let $\alpha$ be a contact structure on a 3-manifold. Then $\mathrm{d}\left(\mathrm{e}^{\theta} \alpha\right)$ is a symplectic form on the 4-dimensional manifold $M \times \mathbb{R}$, where $\theta$ is the coordinate on $\mathbb{R}$. (Here $\alpha$ is written as a form on $M \times \mathbb{R})$.

Proof. We have $\omega_{0}=\mathrm{d}\left(\mathrm{e}^{\theta} \alpha\right)=\mathrm{e}^{\theta}(\mathrm{d} \theta \wedge \alpha+\mathrm{d} \alpha)$. Thus,

$$
\omega_{0} \wedge \omega_{0}=\mathrm{e}^{2 \theta}(2 \mathrm{~d} \theta \wedge \alpha \wedge \mathrm{d} \alpha+\mathrm{d} \alpha \wedge \mathrm{d} \alpha)
$$

Since $\alpha \wedge \mathrm{d} \alpha$ is never zero and since $\mathrm{d} \alpha \wedge \mathrm{d} \alpha$ does not contain differentials of $\theta$, 
the claim follows.

There are also other relations between contact and symplectic geometry [7].

\section{Connections with Riemannian Geometry}

The differentiable structure of a smooth manifold $M$ gives rise to a canonical symplectic form on its cotangent bundle $T^{*} M$. Giving a Riemannian metric $g$ on $M$ is equivalent to prescribing its unit cosphere bundle $S_{g}^{*} M \subset T^{*} M$ and the restriction of the canonical 1-form from $T^{*} M$ gives $S^{*} M$ the structure of a contact manifold.

The following examples of known results are closely related to Riemannian and symplectic aspects of geometry.

1) A submanifold $L$ of a symplectic manifold $(M, \omega)$ is called lagrangian if $\omega=0$ on $T L$.

a) Endow complex projective space $\mathbb{C P}^{n}$ with the usual Kähler metric and the usual Kähler form. The volume of submanifolds is taken with respect to this Riemannian metric. According to a result of Givental-Kleiner-Oh, the standard $\mathbb{R P}^{n}$ in $\mathbb{C P}^{n}$ has minimal volume among all its Hamiltonian deformations [8]. A partial result for the Clifford torus in $\mathbb{C P}^{n}$ can be found in [9]. The torus $S^{1} \times S^{1} \subset S^{2} \times S^{2}$ formed by the equators is also volume minimizing among its Hamiltonian deformations [10]. If $L$ is a closed Lagrangian submanifold of $\left(\mathbb{R}^{2 n}, \omega_{0}\right)$ there exists according to [11] a constant $C$ depending on $L$ such that $\mathrm{Vol}\left(\varphi_{H}(L)\right) \geq C$ for all Hamiltonian deformations of $L$.

b) The mean curvature form of a Lagrangian submanifold $L$ in a Kähler-Einstein manifold can be expressed through symplectic invariants of $L$ [12].

2) To estimate the first eigenvalue of the Laplacian operator on functions for certain Riemannian manifolds, symplectic methods can be used [13].

3) Consider a bounded domain $U \subset \mathbb{R}^{2 n}$ with smooth boundary. There exists a periodic billiard trajectory on $\bar{U}$ of length $/$ with

$$
l^{n} \leq C_{n} \operatorname{vol}(U)
$$

where $C_{n}$ is an explicit constant depending only on $n$ [14].

4) Also Jacobi identity $\{f,\{g, h\}\}+\{h,\{f, g\}\}+\{g,\{h, f\}\}=0$ is satisfied as a consequence of the closure of the symplectic form, $d \omega=0$.

\section{Connections with Kähler Geometry}

Kähler manifolds are the remarkable class of symplectic manifolds. $M$. Gromov [15] observed that some of the tools used in the Kähler context can be used for the study of symplectic manifolds. One part of his wondering work has grown into which is now called Gromov-Witten theory [16]. All Kähler manifolds are symplectic, since the Kähler form is closed and non-degenerate For instance, the complex projective space $\mathbb{C P}^{n}$ is Kähler so that this space is also symplectic. But The converse need not be true, but we have the following theorem:

Theorem 7.1. A structure $(M, \omega, \lambda)$ on a smooth manifold $X$ is a Kähler structure if $\omega$ 
is a symplectic form, $J$ is a complex structure, $g$ is a Riemannian metric such that $g(X, Y)=\omega(X, J Y)$.

Many techniques and constructions from complex geometry are most useful in symplectic geometry. For instance, there is a symplectic version of blowing-up, which is closely related to the symplectic packing problem [17] [18], also Donaldson's construction of symplectic submanifolds [19].

Also any complex surface admits a Kähler structure if and only if the first Betti number is even [20]. There are many symplectic 4-manifolds with even $b 1$ (or $b 1=0)$ admitting no Kähler structure [21]. For a minimal Kähler surface we have the following theorem.

Theorem 7.2 Let $(X, \lambda)$ be a minimal Kähler surface. Then inside the symplectic cone, the Kähler cone can be enlarged across any of its open face determined by an irreducible curve with negative self-intersection. In fact, if the curve is not a rational curve with odd self-intersection, then the reflection of the Kähler cone along the corresponding face is in the symplectic cone.

In addition, for a minimal surface of general type, the canonical class $K_{J}$ is shown to be in the symplectic cone in [22] [23].

\section{Conclusion}

Symplectic geometry is a rather new and vigorously developing mathematical discipline. One can very roughly say that if the fundamental quantity in Riemannian geometry is length, then the fundamental quantity in symplectic geometry is directed area and the fundamental quantity in contact geometry is a certain twisting behavior. In this work, we have developed a connection between various branches of differential geometry with symplectic geometry.

\section{References}

[1] Nazimuddin, A.K.M. and Rifat Hasan, Md. (2014) Applications of Riemannian Geometry Comparing with Symplectic Geometry. Annals of Pure and Applied Mathematics, 6, 170177.

[2] Madsen, I. and Tornehave, J. (1997) From Calculus to Cohomology. Cambridge University Press, Cambridge.

[3] McDuff, D. (1987) Examples of Symplectic Structures. Inventiones Mathematicae, 89, 1336. https://doi.org/10.1007/BF01404672

[4] Eliashberg, Y., Givental, A. and Hofer, H. (2000) Introduction to Symplectic Field Theory. In: Alon, N., et al., Eds., Visions in Mathematics, GAFA 2000 Special Volume, Part II, Birkhäuser, Basel, 560-673.

[5] Hofer, H. (1993) Pseudoholomorphic Curves in Symplectizations with Applications to the Weinstein Conjecture in Dimension Three. Inventiones Mathematicae, 114, 515-563. https://doi.org/10.1007/BF01232679

[6] McDuff, D. and Salamon, D. (1997) Introduction to Symplectic Topology. Clarendon Press, New York.

[7] Eliashberg, Y. (1998) Symplectic Topology in the Nineties. Differential Geometry and Its Applications, 9, 59-88. https://doi.org/10.1016/S0926-2245(98)00018-7 
[8] Oh, Y.-G. (1990) Second Variation and Stabilities of Minimal Lagrangian Submanifolds in Kähler Manifolds. Inventiones Mathematicae, 101, 501-519.

https://doi.org/10.1007/BF01231513

[9] Goldstein, E. (2003) Some Estimates Related to Oh's Conjecture for the Clifford Tori in $\mathbb{C P}^{\mathrm{n}}$. Preprint, math.DG/0311460.

[10] Iriyeh, H., Ono, H. and Sakai, T. (2003) Integral Geometry and Hamiltonian Volume Minimizing Property of a Totally Geodesic Lagrangian Torus in $S^{2} \times S^{2}$. Proceedings of the Japan Academy, Series A, Mathematical Sciences, 79, 167-170. https://doi.org/10.3792/pjaa.79.167

[11] Viterbo, C. (2000) Metric and Isoperimetric Problems in Symplectic Geometry. Journal of the American Mathematical Society, 13, 411-431. https://doi.org/10.1090/S0894-0347-00-00328-3

[12] Cieliebak, K. and Goldstein, E. (2004) A Note on Mean Curvature, Maslov Class and Symplectic Area of Lagrangian Immersions. Journal of Symplectic Geometry, 2, 261-266. https://doi.org/10.4310/JSG.2004.v2.n2.a4

[13] Polterovich, L. (1998) Symplectic Aspects of the First Eigenvalue. Journal für die reine und angewandte Mathematik, 502, 1-17. https://doi.org/10.1515/crll.1998.089

[14] Frauenfelder, U., Ginzburg, V. and Schlenk, F. (2005) Energy Capacity Inequalities via an Action Selector, Geometry, Spectral Theory, Groups, and Dynamics. Contemporary Mathematics, 387, 129-152.

[15] Gromov, M. (1985) Pseudo Holomorphic Curves in Symplectic Manifolds. Inventiones Mathematicae, 82, 307-347. https://doi.org/10.1007/BF01388806

[16] McDuff, D. and Salamon, D. (2004) J-Holomorphic Curves and Symplectic Topology. Vol. 52, American Mathematical Society Colloquium Publications, Providence. https://doi.org/10.1090/coll/052

[17] McDuff, D. (1991) Blowing Ups and Symplectic Embeddings in Dimension 4. Topology, 30, 409-421. https://doi.org/10.1016/0040-9383(91)90021-U

[18] McDuff, D. and Polterovich, L. (1994) Symplectic Packings and Algebraic Geometry. Inventiones Mathematicae, 115, 405-429. https://doi.org/10.1007/BF01231766

[19] Donaldson, S. (1996) Symplectic Submanifolds and Almost-Complex Geometry. Journal of Differential Geometry, 44, 666-705.

[20] Barth, W., Peters, C. and Van de Ven, A. (1984) Compact Complex Surfaces, Ergebnisse der Mathematik und ihrer Grenzgebiete (3) [Results in Mathematics and Related Areas (3)]. Vol. 4, Springer-Verlag, Berlin.

[21] Gompf, R. (1995) A New Construction of Symplectic Manifolds. Annals of Mathematics, 142, 527-595. https://doi.org/10.2307/2118554

[22] Smith, I., Thomas, R. and Yau, S.T. (2002) Symplectic Conifold Transitions. Journal of Differential Geometry, 62, 209-242.

[23] Catanese, F. (2002) Symplectic Structures of Algebraic Surfaces and Deformation. Preprint, Math.AG/0207254. 
Submit or recommend next manuscript to SCIRP and we will provide best service for you:

Accepting pre-submission inquiries through Email, Facebook, LinkedIn, Twitter, etc. A wide selection of journals (inclusive of 9 subjects, more than 200 journals)

Providing 24-hour high-quality service

User-friendly online submission system

Fair and swift peer-review system

Efficient typesetting and proofreading procedure

Display of the result of downloads and visits, as well as the number of cited articles

Maximum dissemination of your research work

Submit your manuscript at: http://papersubmission.scirp.org/

Or contact ajcm@scirp.org 